\title{
Article
}

\section{Towards Anycasting-driven Reservation System for Electric Vehicle Battery Switch Service}

Cao, Yue, Wang, Tong, Zhang, Xing, Kaiwartya, Omprakash, Hashem Eiza, Mahmoud and Putrus, Ghanim

Available at http://clok.uclan.ac.uk/21989/

Cao, Yue, Wang, Tong, Zhang, Xing, Kaiwartya, Omprakash, Hashem Eiza, Mahmoud ORCID: 0000-0001-9114-8577 and Putrus, Ghanim (2019) Towards Anycasting-driven Reservation System for Electric Vehicle Battery Switch Service. IEEE Systems Journal, 13 (1). pp. 906-917. ISSN 1932-8184

It is advisable to refer to the publisher's version if you intend to cite from the work. http://dx.doi.org/10.1109/JSYST.2018.2815156

For more information about UCLan's research in this area go to http://www.uclan.ac.uk/researchgroups/ and search for <name of research Group>.

For information about Research generally at UCLan please go to http://www.uclan.ac.uk/research/

All outputs in CLoK are protected by Intellectual Property Rights law, including Copyright law. Copyright, IPR and Moral Rights for the works on this site are retained by the individual authors and/or other copyright owners. Terms and conditions for use of this material are defined in the policies page.

\section{CLoK}

Central Lancashire online Knowledge www.clok.uclan.ac.uk

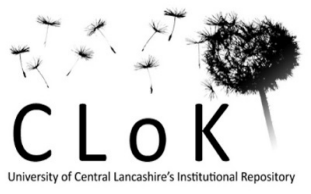




\title{
Towards Anycasting-driven Reservation System for Electric Vehicle Battery Switch Service
}

\author{
Yue Cao, Member, IEEE, Tong Wang, Xing Zhang, Member, IEEE, Omprakash Kaiwartya, Member, IEEE, \\ Mahmoud Hashem Eiza, Member, IEEE and Ghanim Putrus
}

\begin{abstract}
Electro-Mobility has become an increasingly important research problem in urban city. Due to the limited electricity of battery, Electric Vehicle (EV) drivers may experience discomfort for long charging waiting time. Different from plug-in charging technology, we investigate the battery switch technology to improve EV drivers' comfort (e.g., reduce the service waiting time from tens of minutes to a few minutes), by benefiting from switchable (fully-recharged) batteries cycled at Charging Stations (CSs). Since demand hotspot may still happen at CSs (e.g., running out of switchable batteries), incoming EVs may wait additional time to get their battery switched, and thus the EV driver's comfort is degraded. Firstly, we propose a centralized reservation enabling service, considering EVs' reservations (including arrival time, expected charging time of their batteries to be depleted) to optimally coordinate their battery switch plans. Secondly, a decentralized system is further proposed, by facilitating the Vehicle-to-Vehicle (V2V) anycasting to deliver EV's reservations. This helps to address some of the privacy issues that can be materialized in centralized system and reduce communication cost (e.g., through cellular network for reservation making). Results under the Helsinki city scenario show a trade-off between comparable performance (e.g., service waiting time, number of switched batteries) and cellular network cost for EVs' reservations delivery.
\end{abstract}

Index Terms-Electric Vehicle, Transportation Planning, Battery Switch, Anyacsting, Internet of Vehicles.

\section{INTRODUCTION}

$\mathbf{E}$ LECTRIC Vehicles (EVs) [1] are expected to be widely adopted as individual, commercial, and public vehicle fleets. However, compared with traditional gasoline-powered vehicles, EVs are more likely to run out of energy, thus should be charged during their journeys. This is mainly due to the limited EV battery capacity and long trip in big cities (e.g., current battery design only supports EV running with urban area). As a result, how to manage the charging processes of EVs to improve their drivers' comfort, is a vital research issue for the success and long-term viability of EV industry.

Y.Cao, O.Kaiwartya, G.Puturs are with the Department of Computer and Information Sciences, Northumbria University, Newcastle upon Tyne, UK. email: yue.cao; omprakash.kaiwartya; ghanim.putrus@northumbria.ac.uk. T.Wang is with College of Information and Communication Engineering, Harbin Engineering University, China. email: wangtong@hrbeu.edu.cn. X.Zhang is with the School of Information and Communication Engineering, Beijing University of Posts and Telecommunications, China. email: hszhang@bupt.edu.cn. M.Eiza is with the School of Physical Sciences and Computing, University of Central Lancashire. email: mhashemeiza@uclan.ac.uk.

This work was supported in part by the National Natural Science Foundation (61102105, 51779050), Harbin Science Fund for Young Reserve Talents (No. 2017RAQXJ036) and EU - Interreg North Sea Region SEEV4-City projects (J-No.:38-2-23-15).
Majority of previous works investigate "charging scheduling" [1] (concerning when/whether to charge) where EVs have already been parked at homes/Charging Stations (CSs). In contrary, we address "CS-selection" (concerning where to charge) that has not been adequately investigated. In general, public CSs are typically deployed at places where there is high EVs concentration, e.g., shopping mall and parking places. Due to the relatively long time to charge an EV battery, to optimally manage where to charge has become a critical issue in recent years due to the popularization of EVs.

Majority of previous works on CS-selection [2] are generally based on the centralized system. Here, by monitoring CSs' condition, the Global Aggregator (GA) as centralized controller implements the CS-selection decision, whenever it receives a charging request from an $\mathrm{EV}$ on-the-move that needs charging. Several CS-selection schemes [3]-[9] have attempted to minimize the EVs' charging waiting time. Basically, the CS with the highest availability (e.g., minimum queuing time [5]) will be selected as the best choice. Inevitably, a potential charging hotspot may happen, if many EVs travel towards the same CS for charging. If further bringing anticipated EVs' reservations ${ }^{1}$ [10]-[13] (including when the EV will arrive at selected CS for charging, and how long its charging time will be upon the arrival), the congestion at CS could be alleviated. This is because that at what time and which CS will be overloaded can be identified, so as to avoid selecting that CS as the charging plan.

Nevertheless, the plug-in charging technology still requires a relatively longer duration [14] to complete battery charging, thus CSs will be overloaded. The time and efforts spent for seeking available CSs over the city, and waiting in the service queue would bring uncomfortable and anxious driving experience for EV drivers. In contrast to the plug-in charging technology, as a promising alternative approach, the battery switch service [15]-[17], has the potential to replace a fully charged battery for parked EV, just within several minutes. This envisions for an elaborate industrial automation robots to execute fast battery switch.

Even though the centralized system has been proven quite successful in economically scaling and provides optimal allocation, it has own drawbacks. For instance, the failure of

\footnotetext{
${ }^{1}$ Note that, the reservation of EV observed by the GA, will be taken into account for arranging charging plans for other EVs that need the battery switch services in future. The EV's reservation only associates with the CS it has charging intention. If the EV has not been with charging intention, both expected charging time and arrival time can not be resolved, thus no charging reservation will be generated. Note that, the reservation is sent from an EV, only if it has accepted the CS-selection decision from the GA.
} 
GA leads to the service dropout for all EVs drivers. The complexity and computation load of this centralized optimization solution, increases exponentially with the number of EVs. Here, EVs' reservations are generally reported through the conventional ICT technologies, e.g., 4G cellular network. While it is costly and sometime is over-congested, thus causes the degraded communications quality. In this context, a decentralized system is motivated.

Internet of Vehicles (IoV) [18] is one of the revolutions mobilized by Internet of Things (IoT), where the concept of connected vehicle is highly appreciated. The wireless connectivity among EVs creates huge possibilities for sophisticated infotainment systems, application processors, heads-up displays, graphics accelerators, and Vehicle-to-Vehicle (V2V) [19] communications.

In literature, in spite that the battery switch technology has been investigated for "charging scheduling" [16], that effort towards "CS-selection" ${ }^{2}$. Our contributions are as follows:

- Enabling Reservation for Battery Switch Service (Centralized System): In order to minimize the waiting time for battery switch as well as balance the demand load among CSs, we jointly consider the battery switch/charging procedure locally operated at CSs as already taken by [17], and reservations delivered from EVs investigated in this article. Such anticipated information together with the local status of CSs are recorded by the GA, to estimate the future status of CSs (e.g., the expected number of switchable batteries and expected waiting time for switch). The target is to select a CS which will not be highly congested, so as to improve driver's comfort.

- Study of V2V-driven Reservation Delivery (Decentralized System): By transferring from above reservation enabling technology into a decentralized system, we propose a sustainable EV-assisted reservation delivery system to offload the reservations delivery, from the cellular network to IoV (formed by EVs). CSs are set up as Mobile Edge Computing (MEC) [20] servers with information mining, aggregation and sharing of EVs' reservations with each other. Such a feature is deemed as a scalable solution to the long-term introduction of EVs, in terms of communication cost and system scalability.

\section{RELATED WORK}

\section{A. Battery Switch Service}

To promote the popularization of EVs, it is necessary to build the infrastructure for charging batteries. Traditional plug-in recharging is accomplished by plugging the EV into charging slot set at CSs (placed at different city locations). In contrast, at the CSs providing the battery switch service [15], the automated platform switches the depleted batteries from EVs, with a fully charged battery maintained by CSs.

\footnotetext{
${ }^{2}$ Our preliminary work [17] has proposed the first work enabling battery switch for ICT enabling Electro-mobility, study shows the advantage of that over traditional plug-in charging system for CS-selection." [17] has not been adequately made. Further to above motivation for provisioning of battery switch through a decentralized way, there has not been previous work brings the benefit of IoV with anycasting nature for EVs' reservations delivery.
}

The depleted batteries are placed and recharged so that they can be used by other EV drivers. This means that each CS is able to maintain a certain number of batteries for switch. In particular, the battery switch service could be described as a mixture of a drive-through car wash, which normally switches an EV's battery in several minutes, while without requiring the driver to get out of EV.

\section{B. Electro-Mobility For Where to Charge}

In recent few years, the "CS-selection" problem has started to gain interest, from industrial communities thanks to the popularity of EVs. The works in [5], [7], [9] estimate the queuing time at CSs, such that the one with the minimum queueing time is ranked as the best charging option. The work in [3] compares the schemes to select CS based on either the closest distance or minimum waiting time, where results show that the latter performs better given high EVs density under city scenario. In [4], the CS with a higher capability to accept charging requests from on-the-move EVs, will propose this service with a higher frequency, while EVs sense this service with a decreasing function of their current battery levels. The CS-selection scheme in [6] adopts a pricing strategy to minimize congestion and maximize profit, by adapting the price depending on the number of EVs been parked. Game theory strategy [8] is also applicable by balancing the charging plans among EV drivers.

Further to above works just consider local status of CSs, reservation-enabled CS-selection schemes bring anticipated EVs mobility information (reservations) deemed as an additional signalling, in order to estimate whether a CS will be overloaded in a near future. The work in [12] concerns a highway scenario where the EV will pass through all CSs. The expected charging waiting time is calculated for the EV passing through the entire highway, by jointly considering the charging waiting time at a CS where the EV needs charging for the first time and that time spent at subsequent CSs, before exiting the highway. Other works under the plug-in charging service [10], [11], [13] focus on city scenario, where the EV just heads to a single geographically distributed CS for charging. Here, the expected waiting time for charging is associated to that certain CS.

\section{VDTN Anycasting}

The Vehicle Delay/Disruption Tolerant Networks (VDTNs) extend Vehicular Ad hoc NETworks (VANETs) to tolerate communication disruptions in highly mobile situation. In VDTNs, vehicles store and carry network data, while waiting for opportunities to forward it to the destinations. Majority of VDTN routing schemes focus on unicasting (each message is associated with only one destination) and multicasting (the delivery is required by all destination members within a group). Apart from above, anycasting [21] is a service that allows a node to send a message to at least one, and preferably only one of the members in a group. The idea behind anycasting is that a client wants to send messages to any one of several possible servers offering a particular service (but does not care any specific one). Note that in unicasting, 
each data is with a single destination, where there is no such limitation in anycasting. Anycasting can be used to implement resource discovery mechanisms which are powerful building blocks for many distributed systems, including file sharing etc.

\section{Our Contribution}

TABLE I

SUMMARY OF LITERATURE

\begin{tabular}{|c|c|c|}
\hline \multicolumn{3}{|c|}{-Charging Scheduling- } \\
\hline \multicolumn{3}{|c|}{ [1], [16] } \\
\hline & -CS-Selection- \\
\hline & Plug-in Charging & Battery Switch \\
\hline Reservation Not-enabled & {$[2]-[7],[9]$} & {$[8],[17]$} \\
\hline Reservation Enabled & {$[10]-[13]$} & Our Proposed Solution \\
\hline Anycasting Based IoV & N/A & Our Proposed Solution \\
\hline
\end{tabular}

Beyond the literature summarized in TABLE I, we investigate the battery switch technology in this article. This would lead to substantially different design and computation involved for charging management, e.g., how to manage charging and cycling of batteries maintained at the CS side. Upon the battery switch system, we further study the reservation based CSselection policy to guide battery switch plans.

Indeed, using centralized system keeps the edge devices (EV side) simple, and favors more sophisticated centralized optimizations from the GA side based on the aggregated global information. In contrary to centralized system, a much scalable and decentralized system is preferred in a green city scenario, with alleviated privacy concern and less communication cost. In this context, all signallings handled by the GA, will be decoupled between CSs and EVs, through periodical broadcasting and anycasting-driven reservation delivery.

\section{Reservation Enabled Battery Switch Service (CENTRALIZED System)}

\section{A. Network Entities}

Electric Vehicle (EV): Each EV is with a State Of Charge (SOC) threshold. If the ratio between its current energy and maximum energy is below the SOC threshold, the EV starts to negotiate with the GA to find an appropriate CS for battery switch. EV also reports its reservation to the GA, including "at what time it will arrive at the decided CS" and "how long the expected charging time will be for its depleted battery".

Charging Station (CS): It maintains a number of fully charged batteries for switch. Upon the arrival of EVs, the number of maintained (fully charged) batteries will decrease because of switch. These depleted batteries from EVs may have some residual electricity but have not been fully charged yet. Since each CS needs to charge depleted batteries, its number of maintained batteries will increase. The condition information (number of batteries being switchable and being charged) of each CS is monitored by the GA.

Global Aggregator (GA): It is a centralized entity and requires CSs' condition information and EVs' charging reservations for decision making.

\section{B. Assumption}

We consider a city scenario where CSs are geographically deployed in a city. EVs are equipped with wireless communication devices such as 3G/Long Term Evolution (LTE), which allows them to communicate with the GA for request/reply battery switch services. Each CS initially maintains a certain number of fully charged batteries and is with multiple charging slots, such that a number of depleted batteries from EVs can be charged in parallel.

In case of a low electricity stage, an on-the-move EV equipped with GPS navigation would head towards a selected CS (decided by the GA) for the battery switch service. The underlying EV battery switch policy (charging scheduling concerning when/whether to switch a battery to a parked EV) at the CS side, is based on the First Come First Serve (FCFS) order. This means that the parked EV with an earlier arrival time will be scheduled with a higher switch priority. If a CS is fully occupied (meaning it runs out of fully recharged battery for switch), parked EVs need to wait until batteries are switchable. We assume all EVs are with a unique type of battery in this article, further complexity concerning heterogenous batteries is discussed in following section.

\section{System Cycle}

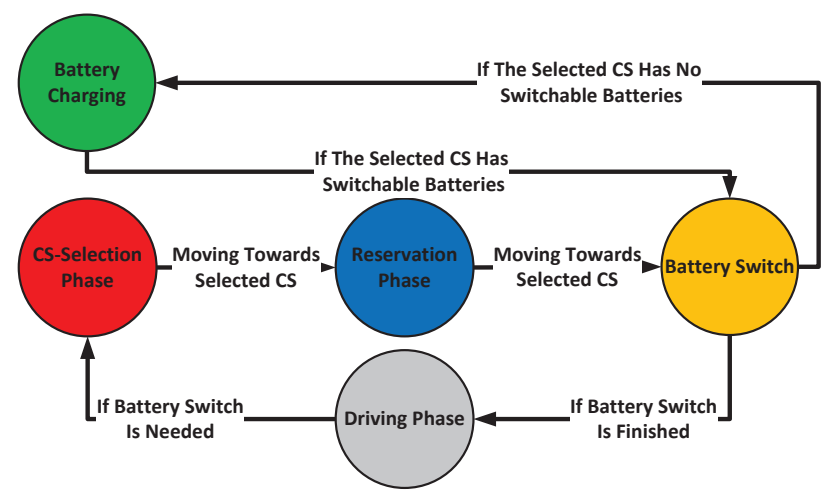

Fig. 1. System Cycle of Proposed EV Charging Management

Fig. 1 describes the cycle of EV charging management:

- Driving Phase: The EV is moving during its routine.

- CS-Selection Phase: The EV reaching a threshold on its residual battery volume, sends its request to the GA, shown in Fig. 2. The GA performs centralized CSselection, and replies the decision back to the EV.

- Reservation Phase: Upon accepting the allocation, the EV further makes its reservation (including its arrival time and expected charging time for its battery) associated with the selected CS, back to the GA.

- Battery Switch Phase: Upon arrival at the selected CS, the EV's battery is switched, with the fully recharged battery maintained at that CS. This happens if the selected CS already maintains a number of fully charged batteries.

- Battery Charging Phase: The batteries depleted from EVs will be charged by CS in parallel (depending on charging slots), and they will be switchable upon being 
fully recharged. The transition between Battery Switch Phase and Battery Charging Phase is bidirectional.

Among them, both the CS-Selection Phase and Reservation Phase are implemented in a centralized manner, because interactions will be handled by the GA.

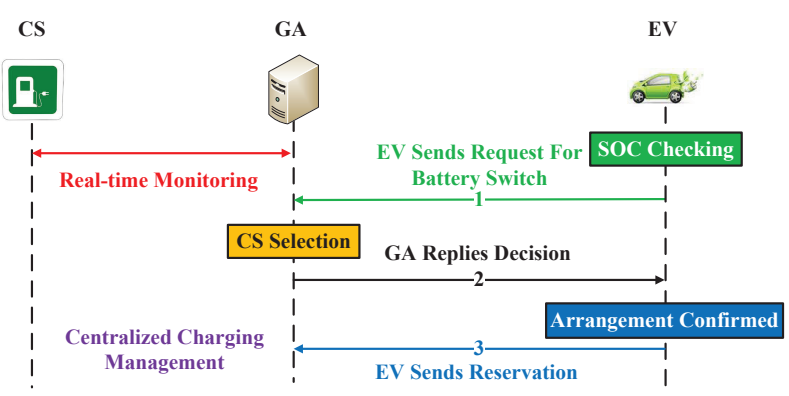

Fig. 2. Example of Centralized EV Charging Management

Fig. 2 shows a typical procedure:

1) The on-the-move EV that needs the battery switch service, namely $\mathrm{EV}_{r}$, informs the $\mathrm{GA}$ about its request.

2) The GA compiles a list of CSs and ranks the most appropriate one (in terms of the balanced charging load among CSs and minimized EV driver's waiting time for the battery switch), replies the CS-decision to $\mathrm{EV}_{r}$.

3) Upon accepting the arrangement, $\mathrm{EV}_{r}$ reports its reservation in relation to selected CS, including its arrival time, expected charging time of its battery upon that arrival.

\section{Battery Management at CS}

1) Battery Switch Procedure: Throughout the battery switch system, we denote as $N_{D}{ }^{3}$ the number of batteries depleted from EVs, and as $N_{C}$ the number of batteries being charged by the CS. Upon arrival at a CS, the incoming EVs need battery switch services are managed as follows:

- If there are switchable batteries at the CS, given by the condition $\left(N_{B}>0\right)$ at line 2 in Algorithm 1, the EV will be directly switched with a fully charged battery.

- Alternatively, presented between lines 4 and 5, the EV has to wait (at the CS) until the recharging of a battery is finished. This is because there has not been any switchable (fully charged) battery available at the CS.

We herein denote as $T_{\mathcal{B}}^{s w}$ the time to switch a battery (normally takes several minutes depending on certain automation technology). Here, the number of switchable batteries $N_{B}$ decreases by 1 , after the period of $T_{\mathcal{B}}^{s w}$ for switch operation. Meanwhile, the depleted battery from EV will be included into the queue of $N_{D}$ (the queue of number batteries waiting to be charged). This refers to the operations between lines 8 and 9 .

\footnotetext{
${ }^{3}$ In other words, $N_{D}$ can be considered as a temporary buffer for depleted batteries from EVs. While CS just processes their charging, with maximum $\delta$ tasks running in parallel, where $\left(N_{C} \leq \delta\right)$.
}

TABLE II

LIST OF NOMENCLATURES

\begin{tabular}{|c|c|}
\hline$\gamma$ & Time interval of system resolution \\
\hline$N_{B}$ & Number of switchable batteries at CS \\
\hline$N_{D}$ & Number of batteries depleted from incoming EVs \\
\hline$T_{\mathcal{B}}^{s w}$ & Time to switch a battery \\
\hline$N_{C}$ & Number of batteries being charged \\
\hline$\delta$ & Number of charging slots at CS \\
\hline$\beta$ & Charging power at CS \\
\hline$E_{\mathcal{B}}^{\text {max }}$ & Full volume of EV battery \\
\hline$E_{\mathcal{B}}^{\text {cur }}$ & Current volume of EV battery \\
\hline ATSLIST & Output list about time available for battery switch \\
\hline$T_{\mathcal{B}}^{\text {fin }}$ & Charging finish time of EV battery \\
\hline$N_{B}$ & Expected number of switchable batteries at CS \\
\hline$\alpha$ & Energy consumption per meter \\
\hline$S_{\text {ev }}$ & EV speed \\
\hline$T_{e v}^{a r r}$ & EV's arrival time at CS \\
\hline$T_{e v}^{t r a}$ & Time for EV to travel towards a CS \\
\hline$T_{c u r}$ & Current time in network \\
\hline$N_{R}$ & Number of EVs made reservations \\
\hline EWTS & Expected waiting time for switch \\
\hline
\end{tabular}

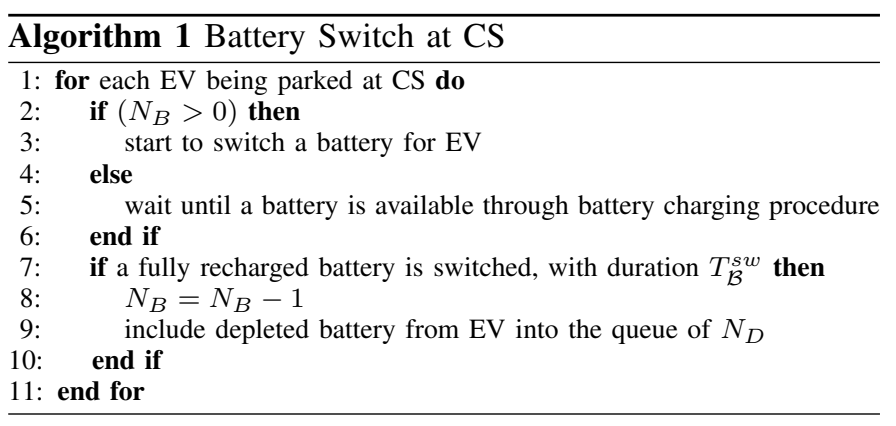

2) Battery Charging Procedure: Note that the CS is with $\delta$ charging slots, meaning that at most $\delta$ depleted batteries can be charged in parallel. As the number of charging slots is normally smaller than number of depleted batteries, depleted batteries are sorted following the Shortest Time Charge First (STCF) order, meaning the depleted battery with the earliest time to be fully charged, has the highest priority for charging. A depleted battery will be scheduled from the queue of $N_{D}$ into the queue of $N_{C}$, only if $\left(N_{C}<\delta\right)$ as presented at line 2 in Algorithm 2. This is due to the availability of charging slots for battery charging.

From line 6 , for each battery in the queue of $N_{C}$, it will be charged with $(\beta \times \gamma)$ electricity per time interval $\gamma$. If a battery is fully recharged given by the condition $\left(E_{\mathcal{B}_{(i)}}^{\text {cur }}=E_{\mathcal{B}_{(i)}}^{\max }\right), N_{B}$ increases by 1 as a fully charged battery is switchable. Then, the information regarding this recently fully charged batteries is removed from the queue of $N_{D}$, at line 10 .

\section{E. Objectives}

We introduce the following notations to facilitate problem formulation of waiting time to perceive battery switch:

- $\gamma_{l_{c s}}$ : Number of EVs currently being parked at a CS, with CS location $l_{c s}$.

- $\omega_{l_{c s}}$ : Average time for each EV to wait for the battery switch (not included the time to switch battery $T_{\mathcal{B}}^{s w}$ ). 


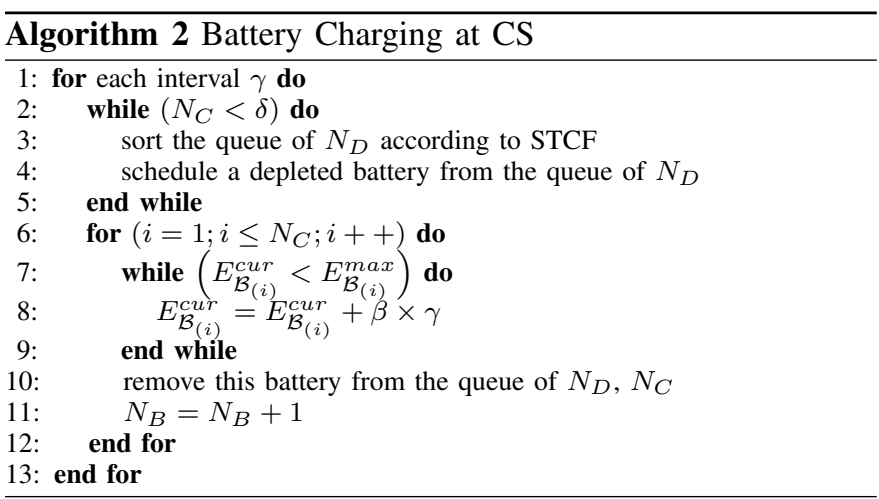

- $\mathcal{W}$ : Total battery switch waiting time for all EVs in network.

Here, note that $\gamma_{l_{c s}}$ is a function of $N_{c s}$, as the number of CSs in network. This is because that a larger number of $N_{c s}$ drives a small $\gamma_{l_{c s}}$ EVs distributed at each CS. Furthermore, $\omega_{l_{c s}}$ is related to $\gamma_{l_{c s}}, \delta$ and $\beta$. Given a number of switchable batteries $N_{B}$, we aim to minimize $\mathcal{W}$ :

$$
\mathcal{W}= \begin{cases}\sum_{l_{c s} \in N_{c s}}\left(\gamma_{l_{c s}} \times\left(\omega_{l_{c s}}+T_{\mathcal{B}}^{s w}\right)\right) & \text { if }\left(N_{B}<\gamma_{l_{c s}}\right) \\ \sum_{l_{c s} \in N_{c s}}\left(\gamma_{l_{c s}} \times\left(0+T_{\mathcal{B}}^{s w}\right)\right) & \text { otherwise }\end{cases}
$$

- The first sub-condition reflects that a larger number of $\gamma_{l_{c s}}$ EVs intend to charge at a CS, inevitably increases their average battery switch waiting time at this CS. Of course, both a fast charging power $\beta$ and more charging slots $\delta$ will reduce such waiting time.

- The second sub-condition implies that $\omega_{l_{c s}}$ tends to 0 , when each CS maintains sufficient number of switchable batteries, given by $\left(N_{B} \geq \gamma_{l_{c s}}\right)$.

As derived in [12] under the plug-in charging system, similarly in order to achieve the minimum waiting time for EVs allocated at $N_{c s}$ CSs under the battery switch system, thereby $\left(\gamma_{l_{c s}} \times\left(\omega_{l_{c s}}+T_{\mathcal{B}}^{s w}\right)\right)$ should be equal among all CSs as ideal situation. Note that under the complex city scenario it is infeasible to achieve the optimal and equal distribution of EVs at all CSs, while our focus is to study the advantage of battery switch system over plug-in charging, upon which we develop a scale and practical reservation solution and evaluate the impact of ICT. Since all CSs share the same $\beta$ and $\delta$, we obtain $\gamma_{l_{c s}}=\mathcal{F}\left(\frac{1}{N_{c s}}\right)$, and $\omega_{l_{c s}}=\mathcal{F}\left(\frac{\gamma_{l_{c s}}}{\delta \times \beta}\right)$ to achieve the minimum $\mathcal{W}$. Also, enabling a large $N_{B}$ is an alternative to minimize $\mathcal{W}$.

In this context, the CS with the highest number of available batteries for switch is selected with the highest priority, in order to hold the second sub-condition. In case that all CSs have run out of batteries for switch, the CS through which an EV experiences the minimum time to wait for the battery switch service is selected. Our proposed CS-selection indeed follows above discussion, the following evaluation results will address all factors involved herein.

\section{F. Reservation Enabled CS-Selection}

At the GA side, the decision making on where to switch battery, considers those anticipated EVs' reservation information as well as availability of CS to provide battery switch service.

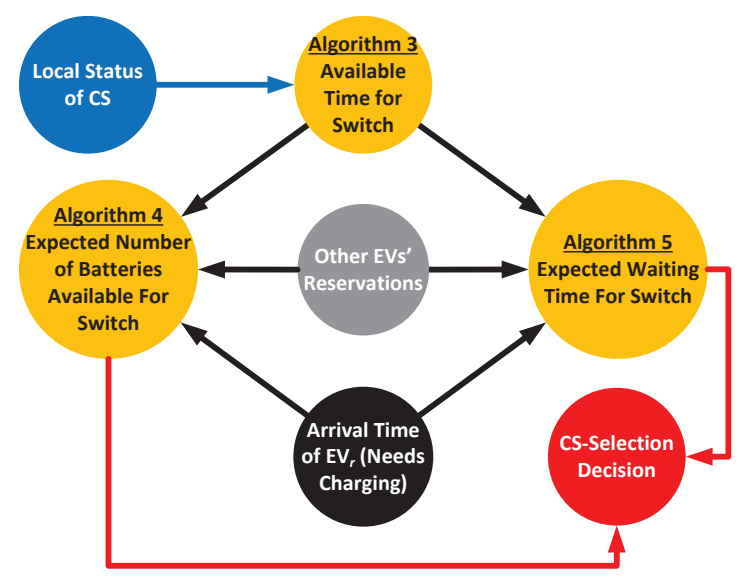

Fig. 3. Flow Chart of Computation Logic

With the knowledge about the EV's reservations as well as local status of CS, both the expected number of batteries available for switch (as denoted by $\overline{N_{B}}$ ), and Expected Waiting Time for Switch (EWTS) at a CS can be estimated.

The CS-selection aims to reduce the average EV driver's perceived waiting time at CS, meanwhile balance the load among CSs. In special case, EV driver may need to wait for additional time, in case with the unavailability of batteries at a CS. Following Section III-E, we have:

- First, to select the CS with the maximum value of $\overline{N_{B}}$ from all CSs.

- Second, if all CSs are not eligible to provide the battery switch services (means none of them has switchable battery), the one with the minimum EWTS is selected.

The entire logic is illustrated in Fig. 3. The available time for battery switch at a CS is estimated based on its local condition, as detailed in Algorithm 3. Upon this, those incoming EVs' reservations are jointly considered to estimate the future status of CS. Here, we refer to the future status as expected number of batteries available for switch (as detailed in Algorithm 4), and EWTS (as detailed in Algorithm 5).

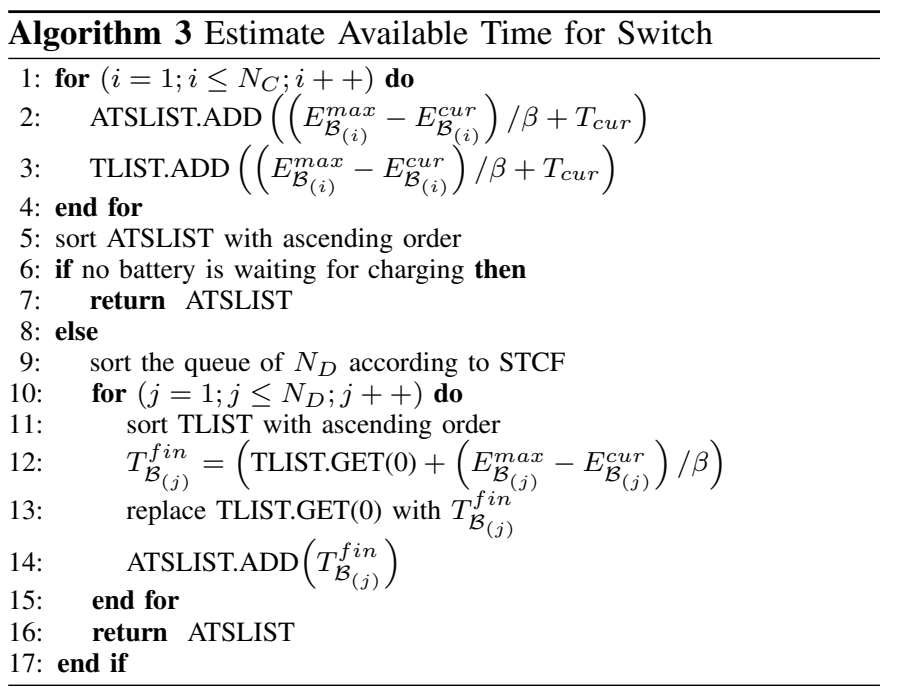

1) Estimate Available Time for Switch: For estimating the available time for a fully charged battery at a CS, we consider 
two types of queues. Those batteries which are under charging are characterized in the queue of $N_{C}$, while those still waiting for charging are characterized in the queue of $N_{D}$.

Algorithm 3 starts from processing each charging battery (in the queue of $N_{C}$ ), where its time duration $\left(E_{\mathcal{B}_{(i)}}^{\max }-E_{\mathcal{B}_{(i)}}^{\text {cur }}\right) / \beta$ to be fully recharged will be summated with $T_{\text {cur }}$. This summated value is as the charging finish time of battery, and then it is included into ATSLIST (as monitored by the GA) and TLIST (for computation purpose), presented at lines 2 and 3.

Upon above processing for those batteries under charging, Algorithm 3 will return the ATSLIST, if the number of batteries waiting for charging is 0 as the condition stated at line 6 , or a loop operation for each battery waiting for charging has been processed (as stated between lines 10 and 16).

In the latter case, the loop operation starts by sorting the queue of $N_{D}$, based on the SCTF charging scheduling order. Meanwhile, the TLIST containing when the charging of those batteries (in the queue of $N_{C}$ ) will be finished, is initialized with an ascending order. Therefore, the earliest available time is at the head of TLIST, denoted by TLIST.GET(0).

Within each loop, the charging finish time $T_{\mathcal{B}_{(i)}}^{\text {fin }}$ of each battery (in the queue of $N_{D}$ ) will replace with TLIST.GET(0). At line $12, T_{\mathcal{B}_{(j)}}^{f i n}$ is calculated as the summation of time to start charging as denoted by TLIST.GET(0), and battery charging time given by $\left(E_{\mathcal{B}_{(j)}}^{\max }-E_{\mathcal{B}_{(j)}}^{\text {cur }}\right) / \beta$. Furthermore, $T_{\mathcal{B}_{(j)}}^{\text {fin }}$ will be included into ATSLIST.

Above loop operation ends when all batteries (in the queue of $N_{D}$ ) have been processed, then the ATSLIST is returned. By recursing Algorithm 3 for each CS, their available time for switch can be estimated by the GA.

2) Reporting Reservation Information: Whenever a CSselection decision is made and returned to the $\mathrm{EV}_{r}$ (the $\mathrm{EV}$ needs the battery switch service) which sent request to the $\mathrm{GA}$, the following information together with the IDs of EV and the selected CS will be reported to the GA, as the EV's reservation information, given by an example in TABLE III.

TABLE III

EV RESERVATION INFORMATION

\begin{tabular}{|l|l|l|l|}
\hline EV ID & Selected CS ID & Arrival Time & Expected Charging Time \\
\hline
\end{tabular}

Arrival Time: We denote $T_{e v}^{a r r}$ as the time slot during which an EV will arrive at the selected CS, where:

$$
T_{e v}^{a r r}=T_{c u r}+T_{e v}^{t r a}
$$

Here, $T_{e v}^{\text {tra }}$ is the travelling time measured from the current location of EV to the selected CS, via the shortest road path. Note that $T_{\text {cur }}$ is the current time in network.

Expected Charging Time: We denote as $T_{\mathcal{B}}^{c h a}$ the expected charging time of the EV's depleted battery upon that arrival, where:

$$
T_{\mathcal{B}}^{c h a}=\frac{E_{\mathcal{B}}^{\max }-E_{\mathcal{B}}^{c u r}+S_{e v} \times T_{e v}^{t r a} \times \alpha}{\beta}
$$

Here, $\left(S_{e v} \times T_{e v}^{t r a} \times \alpha\right)$ is the energy consumed for the movement travelling to the selected CS, based on a constant $\alpha$ (depending on a certain type EV) measuring the energy consumption per meter. Therefore, $\left(E_{\mathcal{B}}^{\max }-E_{\mathcal{B}}^{c u r}+S_{e v} \times T_{e v}^{\text {tra }} \times \alpha\right)$ is the expected electricity of the battery (will be depleted from that EV upon arrival) needs to be recharged, depending on the charging power $\beta$ of CS.

The assumption of trustworthy reservation, is vulnerable without ensuring the integrity of messages from EVs to the GA on end-to-end aspects. E.g., forged or wrong reservation information are continuously delivered by the GA to compute quite imprecise estimation for charging waiting time. The general secured vehicular communication framework in [22] can be applied to enable secured delivery of EVs' reservation. Besides, in the case of uncertain EV arrival [13] due to traffic jam, it will also be of importance to periodically update EV's reservation to GA, such that a revised decision could be recommended to EV. In such a case, EV may change the plan to switch the battery at original CS and head to the CS subjects to revised decision.

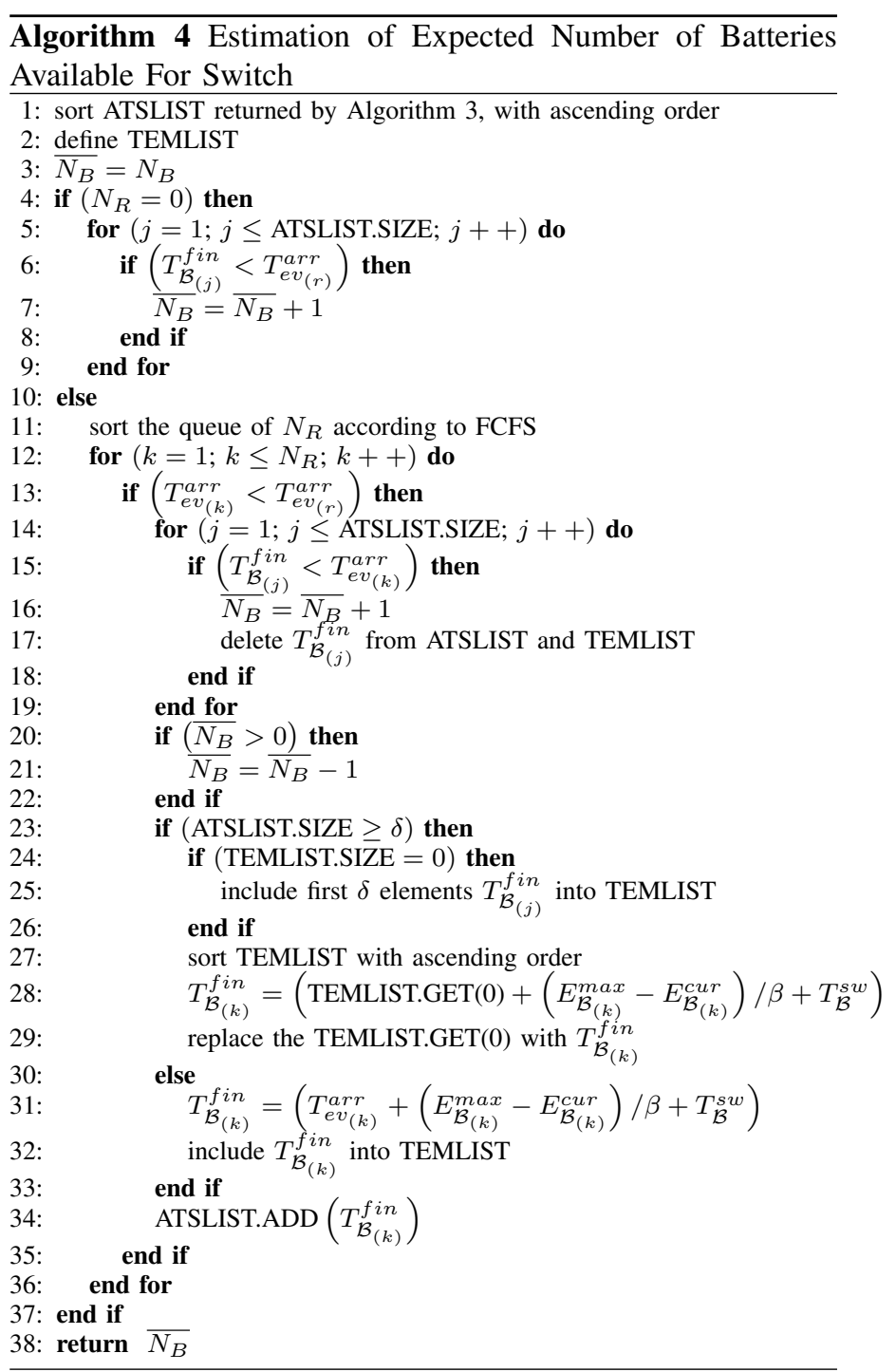

3) Estimate Expected Number of Batteries Available For Switch: Algorithm 4 presents the detail to estimate the expected number of batteries available for switch, as denoted by $\overline{N_{B}}$. As indicated in Fig. 3, it also requires the knowledge of available time for battery switch from Algorithm 3, as presented at line 1 . Here, we denote as $N_{R}$ the number of 
EVs have already made reservations for the battery switch at the CS, and initialize $\overline{N_{B}}$ with the value of $N_{B}$.

In special case that the CS is not reserved by any $\mathrm{EV}$, as given by the condition $\left(N_{R}=0\right)$ at line 4 , the arrival time of $\mathrm{EV}_{r}$, as $T_{e v_{(r)}}^{a r r}$ is compared with the charging finish time of each battery (being charged or waiting to be charged) at this CS. If any $T_{\mathcal{B}_{(j)}}^{f i n}$ is earlier than $T_{e v_{(r)}}^{a r r}$, this means one more battery will be available for switch upon the arrival of $\mathrm{EV}_{r}$, with $\bar{N}_{B}$ increases by 1 as presented at line 7 . Also, the given $T_{\mathcal{B}_{(i)}}^{\text {fin }}$ will be removed from ATSLIST, meaning the number of batteries (being charged or waiting to be charged) decreases.

Then Algorithm 4 sorts the queue of $N_{R}$ following FCFS order, which is same as the charging scheduling priority upon EVs arrival. In this case, $\mathrm{EV}_{k}$ stands for the $k^{t h} \mathrm{EV}$ in the queue of $N_{R}$. Normally, the arrival time $T_{e v_{(k)}}^{a r r}$ of each $\mathrm{EV}_{k}$ (in the queue of $N_{R}$ ) made reservation at the CS, will be compared with $T_{e v_{(r)}}^{a r r}$ (the arrival time of $\mathrm{EV}_{r}$ ). As highlighted at line 13, for each $T_{e v(k)}^{a r r}$ which is earlier than $T_{e v(r)}^{a r r}$, the former will involve the dynamic update of ATSLIST. This reflects only those EVs (in the queue of $N_{R}$ ) with an earlier arrival time than $\mathrm{EV}_{r}$, are considered for calculating $\overline{N_{B}}$.

Note that the ATSLIST has been initially sorted according to the ascending order, such that the earliest available time for switch is at the head of ATSLIST. From line 15, $T_{e v(k)}^{a r r}$ is compared with the charging finish time of each battery (being charged or waiting to be charged) at this CS. If $T_{\mathcal{B}_{(j)}}^{f i n}$ is earlier than $T_{e v_{(k)}}^{a r r}$, one more battery will be switchable upon the arrival of $\mathrm{EV}_{k}$, with $\overline{N_{B}}$ increases by 1, as presented at line 16 . As such, the given $T_{\mathcal{B}_{(j)}}^{\text {fin }}$ will be removed from ATSLIST (and also TEMLIST initialized from line 24), meaning the number of batteries being charged or to be charged decreases.

At line 21, the number of switchable batteries decreases by 1 , as $\mathrm{EV}_{k}$ will be replaced with a fully charged battery. Then:

- As given by the condition (ATSLIST.SIZE $\geq \delta$ ) at line 23 , if the number of batteries being charged or to be charged, is larger than the total number of charging slots a CS is equipped, this reflects any incoming $\mathrm{EV}_{k}$ still needs to wait for additional time until a fully recharged battery is available for switch. In this case, the charging finish time $T_{\mathcal{B}_{(k)}}^{\text {fin }}$ of the battery depleted from $\mathrm{EV}_{k}$ is given at line 28:

$$
T_{\mathcal{B}_{(k)}}^{\text {fin }}=\left(\text { TEMLIST.GET }(0)+\left(E_{\mathcal{B}_{(k)}}^{\text {max }}-E_{\mathcal{B}_{(k)}}^{\text {cur }}\right) / \beta+T_{\mathcal{B}}^{s w}\right)
$$

where TEMLIST.GET $(0)^{4}$ is the time when a charging slot is available at the CS, $\left(E_{\mathcal{B}_{(k)}}^{\max }-E_{\mathcal{B}_{(k)}}^{\text {cur }}\right) / \beta$ is the time to fully recharge the battery depleted from $\mathrm{EV}_{k}$, while $T_{\mathcal{B}}^{s w}$ is the time duration to deplete this battery from $\mathrm{EV}_{k}$ and switch it with a fully recharged battery.

- Otherwise, $\mathrm{EV}_{k}$ can be directly switched with a fully recharged battery without waiting, with $T_{\mathcal{B}_{(k)}}^{f i n}$ given at

\footnotetext{
${ }^{4}$ As we also define TEMLIST at line 2, the first $\delta$ value in ATSLIST are included into TEMLIST. This certainly reflects the charging finish time of batteries being charged at CS. At line 29 , replacing $T_{\mathcal{B}_{(k)}}^{\text {fin }}$ with TEMLIST.GET(0) thus updates the charging finish time of batteries in TEMLIST, for the computation that $\mathrm{EV}_{k}$ involves in next loop.
}

line 31:

$$
T_{\mathcal{B}_{(k)}}^{\text {fin }}=\left(T_{\text {ev }(k)}^{a r r}+\left(E_{\mathcal{B}_{(k)}}^{\max }-E_{\mathcal{B}_{(k)}}^{\text {cur }}\right) / \beta+T_{\mathcal{B}}^{s w}\right)
$$

Note that the time to start battery switch is $T_{e v_{(k)}}^{a r r}$, as the arrival time of EV.

Furthermore, the charging finish time of each battery depleted from incoming $\mathrm{EV}_{k}$, will be included into ATSLIST at line 34. This procedure is repeated, until all $\mathrm{EV}_{k}$ (in the queue of $N_{R}$ ) have been processed. Finally, the expected number of batteries available for switch $\overline{N_{B}}$ is given at line 38 .

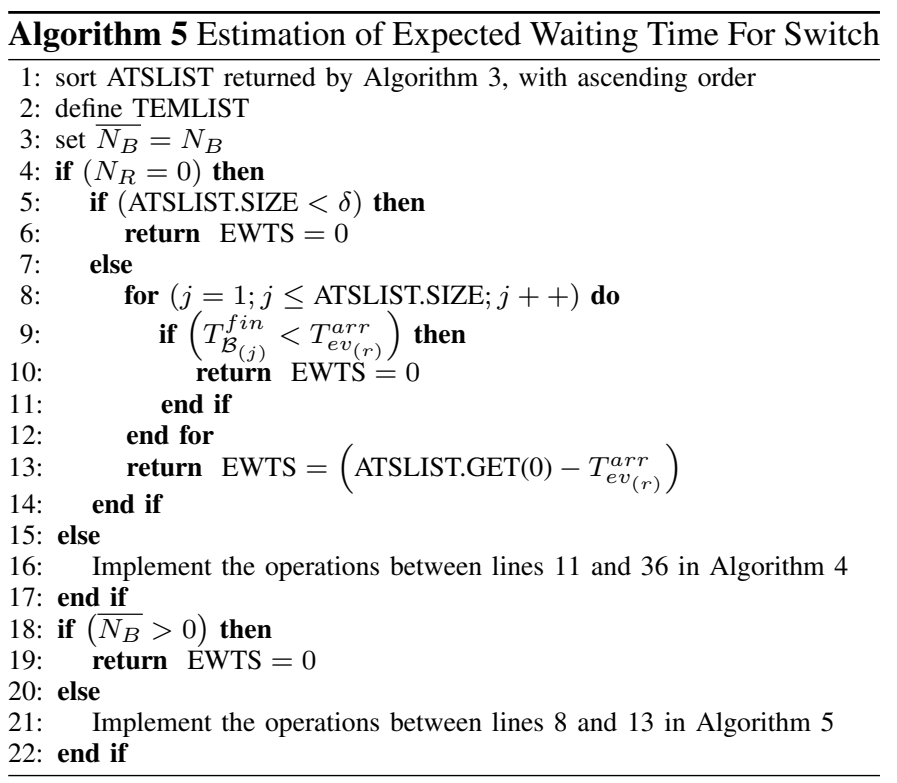

4) Estimate Expected Waiting Time For Switch: Similar to Algorithm 4, Algorithm 5 which presents the detail to estimate the Expected Waiting Time for Switch (EWTS) also requires the knowledge from Algorithm 3 as well as those EVs making reservations. This provides a way to estimate the $\omega_{l_{c s}}$ as discussed in Section III-E.

In special case that there has not been any EV made reservation at the CS, the EWTS is only related to the local status of CS. Here, $T_{e v_{(r)}}^{a r r}$ is compared with the charging finish time $T_{\mathcal{B}_{(j)}}^{\text {fin }}$ of each battery (being charged or waiting to be charged) at this CS, specifically:

- If there is any $T_{\mathcal{B}_{(j)}}^{f i n}$ earlier than $T_{e v(r)}^{a r r}$, this means one more battery will be available for switch upon the arrival of $\mathrm{EV}_{r}$. As such, the EWTS is returned as 0 at line 10 , since incoming EV will not experience any delay to wait for a switchable battery. Additionally, if the size of ATSLIST is smaller than value of charging slots as given by (ATSLIST.SIZE $<\delta$ ), the EWTS is returned as 0 at line 6 , as charging slots are not fully occupied (the CS can fully charge $\delta$ batteries).

- Otherwise, the EWTS is returned as (ATSLIST.GET $(0)-T_{e v(r)}^{a r r}$ ) at line 13, if there has not been any battery available for switch upon the arrival of $\mathrm{EV}_{r}$. Here, ATSLIST.GET(0) is the earliest time to get a switchable battery. 
From line 16, each EV (in the queue of $N_{R}$ ) made reservation will be processed, by following the same operations between lines 11 and 36 in Algorithm 4. This mainly involves into the update of ATSLIST and $\overline{N_{B}}$, depending on participated EVs reservations information. Above procedure is repeated until all $\mathrm{EV}_{k}$ (in the queue of $N_{R}$ ) have been processed, finally:

- Presented between lines 18 and 19, the EWTS is returned as 0 if $\overline{N_{B}}$ is still larger than 0 , since there is no waiting time to experience the battery switch service. This is also same as the case if the arrival time of $\mathrm{EV}_{r}$ as $T_{e v_{(r)}}^{a r r}$, is later than the earliest time a battery is switchable, presented between lines 9 and 10 .

- Alternatively, EWST is given by the rule at line 21, following the same operation between lines 8 and 13 in Algorithm 5. This determines whether there is a switchable battery upon the arrival of $\mathrm{EV}_{r}$, by comparing each $T_{\mathcal{B}_{(j)}}^{f i n}$ in ATSLIST with $T_{e v_{(r)}}^{a r r}$.

\section{Reservation Enabled Battery Switch Service (DECENTRALIZED SySTEM)}

\section{A. Privacy Concern in Centralized System}

In general, the battery switch service can be executed in both centralized and distributed manners. With the centralized manner, the CS-selection is executed by the GA, as presented in Section III-F. However, this arises much privacy concern, because the EV status information (e.g., location, ID) needs to be released. In contrary, the decentralized manner benefits from a low privacy sensitivity, where the CS-selection decision is executed by EV individually (using the information broadcasted from CSs). Importantly, the accuracy of information (ATSLIST calculated in Algorithm 3, $N_{B}$ and associated EVs' reservations formatted in TABLE III) plays an important role in CS-selection, particularly in decentralized manner. This is because that the CS-selection decision would be suboptimal, due to obsolete information involved for CS-selection.

\section{B. Communication Signallings}

TABLE IV

FORMAT OF CS BROADCASTING

\begin{tabular}{|c|c|c|}
\hline & & -CS ID- \\
\hline & & $\mathrm{CS}_{1}$ \\
\hline & -Numbe & of Switchable Batteries- \\
\hline & & $N_{B}=3$ \\
\hline & -Avai & ble Time For Switch- \\
\hline & ATSLIS & $=[2000 \mathrm{~s}, 3400 \mathrm{~s}, 3900 \mathrm{~s}]$ \\
\hline & -Anony & ous EVs' Reservations- \\
\hline Entry & Arrival Time & Expected Charging Time of Depleted Battery \\
\hline 1 & $3300 \mathrm{~s}$ & $300 \mathrm{~s}$ \\
\hline ..... & $\ldots .$. & $\ldots .$. \\
\hline 6 & $4700 \mathrm{~s}$ & $700 \mathrm{~s}$ \\
\hline
\end{tabular}

Motivated by the concern on privacy, we propose a decentralized system (without GA involved for handling optimization), where non-realtime information is exchanged between CSs and EVs. Major differences from Section III-F are on
CS-selection Phase and Reservation Phase. In decentralized system, each CS broadcasts its information formatted in TABLE IV, to EVs through the cellular network, and acquires its associated EV's reservations (primarily through IoV anycasting, and additionally cellular network as the backup). Fig. 4 illustrates a typical procedure:

1) Each CS periodically (with interval $\Delta$ ) broadcasts its information throughout the cellular network. Thus, each EV in network can always access broadcasted information from CSs, within interval $\Delta$.

2) The EV which has planned on where to charge, namely $\mathrm{EV}_{r}$, reports its reservation to its selected CS. The reservation could be relayed by any encountered EV, namely $\mathrm{EV}_{x}$ to a CS. Here, $\mathrm{EV}_{x}$ is qualified by whether it can help with delivery before the time slot $(\Delta+L)$ (as the time slot of next CSs broadcasting) ${ }^{5}$, where $L$ is the previous broadcasting time slot.

3) The V2V anycasting will be repeated, until the reservation of $\mathrm{EV}_{r}$ is finally delivered to a CS. This refers to a "one-to-any" paradigm, as the delivery ends up at any one of CSs (does not need to be the CS selected by $\mathrm{EV}_{r}$ ). Here, an acknowledge of successful reservation making will be replied to $\mathrm{EV}_{r}$ (omitted in signalling procedure).

4) Each CS analyzes and mines valid information from delivered EVs' reservations. The valid information refers to those reservations of which the EV's arrival is supposed to be later than the $(\Delta+L)$. Such mined reservations will be aggregated and further exchanged among CSs through Internet, depending on the ID of CS (selected by the EVs with common charging intentions). As an example in Fig. 5, aggregated EVs' reservations associated to $\mathrm{CS}_{3}$ (delivered by $\mathrm{CS}_{1}$ through $\mathrm{V} 2 \mathrm{~V}$ anycasting), will be sent to $\mathrm{CS}_{3}$ through Internet. Then, at the time slot approaching $(\Delta+L)$ :

- Each CS merges its associated EVs' reservations with its local condition (ATSLIST and $N_{B}$ ) for broadcasting, following the format of TABLE IV.

- If the reservation of $\mathrm{EV}_{r}$ has not been delivered through $\mathrm{V} 2 \mathrm{~V}$ anycasting (e.g., $\mathrm{EV}_{r}$ has not received acknowledgement from its planned CS), then $\mathrm{EV}_{r}$ directly reports its reservation to the selected CS through the cellular network.

\section{Analysis on Communication Cost}

1) Decentralized System: Each CS experiences a communication cost of $O\left(\frac{N_{e v}}{\Delta}\right)$, for broadcasting its information (ATSLIST calculated in Algorithm 3, $N_{B}$ and associated EVs' reservations formatted in TABLE III) to all EVs. Here, $N_{e v}$ is the number of EVs. The situation for reservation making depends on following options:

- If with the $\mathrm{V} 2 \mathrm{~V}$ anycasting for reservations delivery to any CS, such a way experiences a cost of $O\left(N_{e v}\right)$, depending on EVs density. Of course, to appropriately

\footnotetext{
${ }^{5}$ We consider all CSs' broadcasting is synchronized, such system is also applicable to the case where CSs are with different broadcasting intervals.
} 


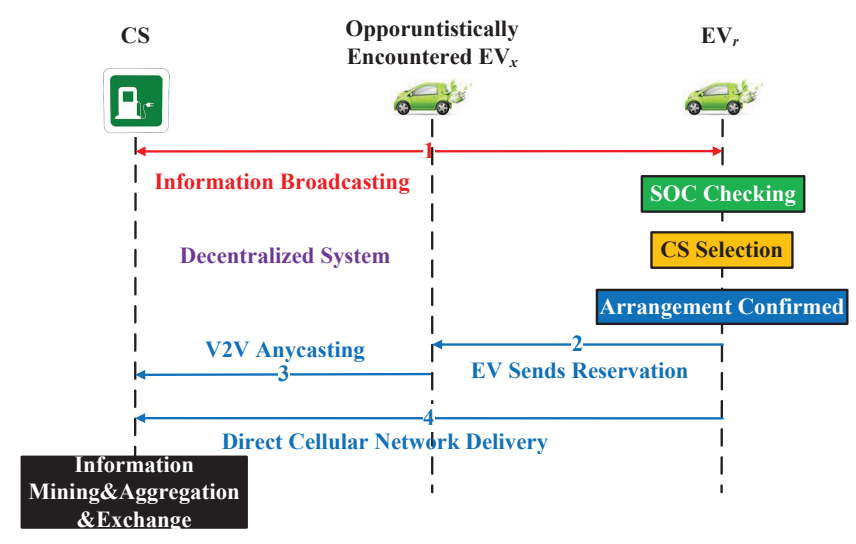

Fig. 4. Communication Signallings

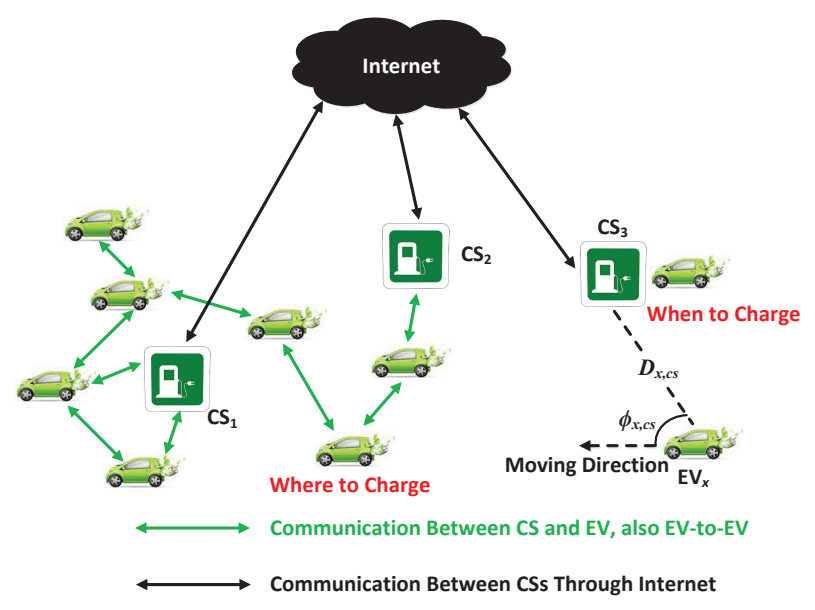

Fig. 5. Big Picture of Decentralized System

select a small number of EVs as relays would further reduce the cost, as widely studied in DTN routing [21].

- Note that, the cellular network is adopted as the backup solution by $\mathrm{EV}_{r}$, only if its reservation has not been delivered at the time slot approaching $(\Delta+L)$. Here, $\mathrm{EV}_{r}$ will wait for a certain time than use the cellular network, if it doesn't receive a confirmation. As such, the system experiences a cost of $O(\mathcal{R})$, where $\mathcal{R}$ is directly related to the number of battery switch requests.

2) Centralized System: The cost at the GA side for handling EVs' charging requests and reservations are both $O(\mathcal{R})$.

3) Decentralized v.s. Centralized System: In reality, it is reasonable to meet $\left(\mathcal{R} \geq N_{e v}\right)$, which means that each EV needs to charge more than once in the long term. Thus we claim that the communication efficiency of decentralized system, for sustainable delivery of reservations. This is achieved by transferring the communication cost from density of service requests $\mathcal{R}$, to the density of EVs $N_{e v}$.

\section{Reservation Delivery Intelligence}

We assume EVs adopt pseudonyms scheme so that their real IDs won't be revealed or known to other vehicles. This is important to make sure the CS can also verify the received requests as legitimate. Otherwise, attackers could overload the
CS with fake requests causing Denial of Service (DoS) attack. As illustrated in Fig. 6, EVs' reservations are delivered through the following three options in decentralized system:

1) Vehicle-Assisted Direct Delivery: If the encountered EV (namely $\mathrm{EV}_{x}$ ) is also travelling towards its selected CS (with its arrival time $T_{e v}^{a r r}$, which does not need to be the same CS selected by $\mathrm{EV}_{r}$ ), we have:

$$
\left(T_{e v(r)}^{a r r} \geq(\Delta+L)\right) \text { and }\left(T_{e v_{(x)}}^{a r r}<(\Delta+L)\right)
$$

to trigger $\mathrm{EV}_{r}$ to replicate a copy of its reservation to $\mathrm{EV}_{x}$. This is because the reservation from $\mathrm{EV}_{r}$ is only useful ${ }^{6}$ to predict the future status of the CS (where $\mathrm{EV}_{r}$ intends to charge), given by $\left(T_{e v_{(r)}}^{a r r} \geq(\Delta+L)\right)$. As such, to timely deliver the reservation of $\mathrm{EV}_{r}$ bounded by $(\Delta+L)$, is facilitated by a faster mobility of $\mathrm{EV}_{x}$, with $\left(T_{e v(x)}^{a r r}<(\Delta+L)\right)$.

2) Opportunistic V2V Anycasting: If $\mathrm{EV}_{x}$ has not been in charging planning towards its selected CS, a DTN based anycasting scheme is applied. To estimate the delivery potential of $\mathrm{EV}_{x}$, we denote the anycast probability to deliver the reservation of $\mathrm{EV}_{r}$, to any one of $N_{c s}$ CSs, as $\mathcal{P}$ :

$$
\mathcal{P}=1-\left(1-P_{c s}\right)^{N_{c s}}
$$

Here, $\left(1-P_{c s}\right)$ means the probability the reservation is not delivered, while $P_{c s}$ is the successful probability of this event.

We propose a geo-centric anycasting approach based on Equation (7), by concerning speed $S_{x}$, a relative moving direction towards a CS $\phi_{x, c s}$, and distance $D_{x, c s}$ between $\mathrm{EV}_{x}$ and a CS (shown in Fig. 5). To qualify $P_{c s}$ bounded by $(\Delta+L)$, we further define $\left(H=\Delta+L-T_{\text {cur }}\right)$ as the remaining time left to that time bound $(\Delta+L)$, where $T_{\text {cur }}$ is the current time in network.

Next, we apply our previous work, a unicasting routing scheme Delegation Geographic Routing (DGR) [19] to the EV charging use case. It utilizes $\frac{D_{x, c s}-T}{\phi_{x, c s} \times S_{x}}$ as the intersect time to $\mathrm{CS}$, where $T$ is the $\mathrm{V} 2 \mathrm{~V}$ communication radius (also for that between EV and CS). Then we have:

$$
P_{c s}= \begin{cases}\frac{H-\frac{D_{x, c s}-T}{\phi_{x, c s} \times S_{x}}}{H} & \text { if }\left(\phi_{x, c s}<\frac{\pi}{2}\right) \text { and }\left(H>\frac{D_{x, c s}-T}{\phi_{x, c s} \times S_{x}}\right) \\ 0 & \text { else }\end{cases}
$$

Depending on the mobility of $\mathrm{EV}_{x}$, the more CSs it can intersect with forwarding progress $\left(\phi_{x, c s}<\frac{\pi}{2}\right)$ and earlier arrival than $H$, the higher $\mathcal{P}$ will be. Further to this, an iterative optimization [19] for fast converged routing decision is implemented to reduce the communication cost involved for V2V manner to $O\left(\sqrt{N_{e v}}\right)$ (e.g., not select $\mathrm{EV}_{x}$ if it does not extensively contribute to delivery).

3) Direct Cellular Network Reporting: $\mathrm{EV}_{r}$ would switch to the cellular network, for reporting its reservation to the selected CS. This happens at the time slot approaching $(\Delta+$ $L$ ), while the reservation has not been delivered through above two options. 


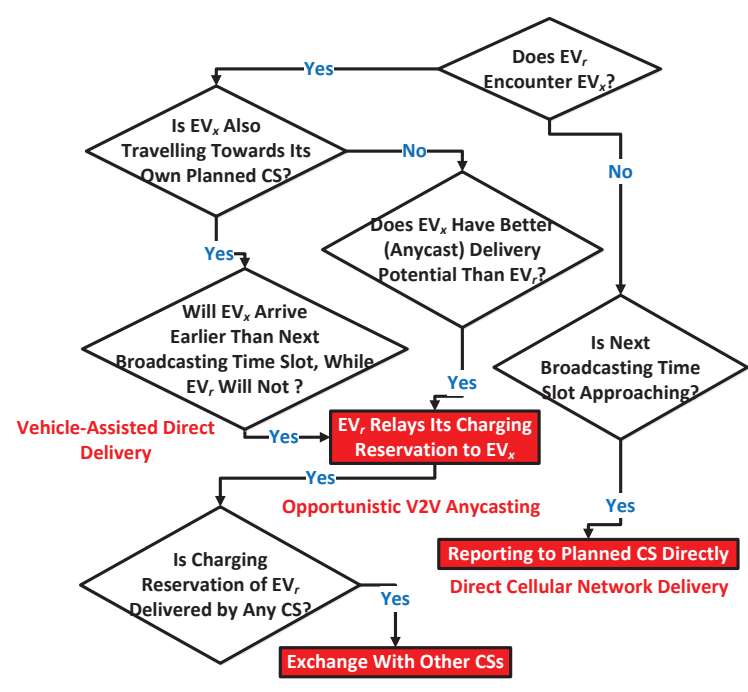

Fig. 6. Flow Chart of Reservation Delivery

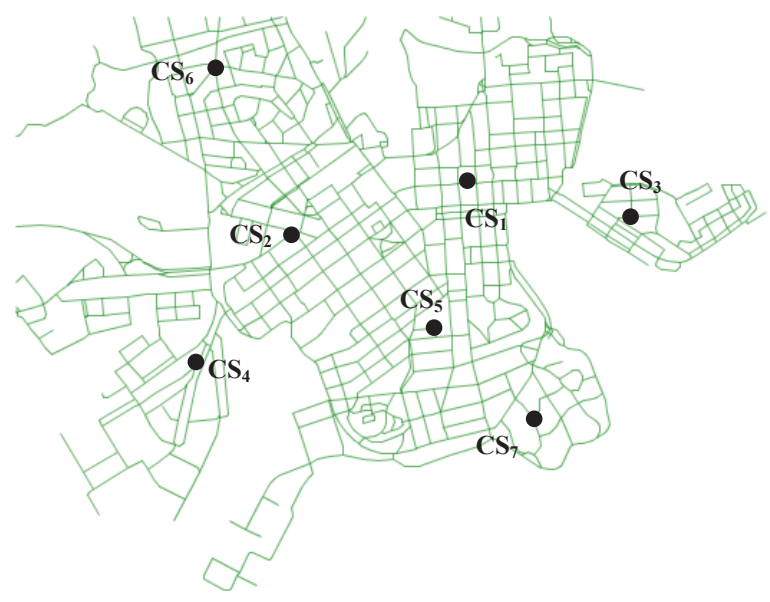

Fig. 7. Simulation Scenario of Helsinki City

\section{Performance Evaluation}

We have built up an entire EV charging system in Opportunistic Network Environment (ONE) [23], the ONE was a network simulator developed for VANETs communication. In Fig. 7, the default scenario with $4500 \times 3400 \mathrm{~m}^{2}$ area is shown as the down town area of Helsinki city abstracted from Google map. Here, $300 \mathrm{EVs}$ with $[30 \sim 50] \mathrm{km} / \mathrm{h}$ variable moving speed and $300 \mathrm{~m}$ transmission range are initialized in the network. The destination of each EV trip is randomly selected from a location in the map. Particularly, once the current destination is reached, a new destination is randomly chosen again. Such procedure is repeated until the EV reaches the SOC threshold, and then requests the battery switch service. The configuration of EVs follows the charging specification \{Maximum Electricity Capacity (MEC), Max Travelling Distance (MTD), SOC threshold\}. EVs are with the type of Hyundai BlueOn as set in [13] $\{16.4 \mathrm{kWh}, 140$ $\mathrm{km}, 15 \sim 45 \%$ \}.

\footnotetext{
${ }^{6}$ The charging reservation of $\mathrm{EV}_{r}$ with an earlier arrival than $(\Delta+L)$, will not be mined by CSs for future broadcasting. This is because the reservation of $\mathrm{EV}_{r}$ will be deleted by its selected CS, upon once being parked at there.
}

Here, the electricity consumption for the Traveled Distance (TD) is calculated based on $\frac{\mathrm{MEC} \times \mathrm{TD}}{\mathrm{MTD}}$, as widely used in literature such as [9]. All EVs' batteries are with full volume at beginning. Besides, 7 CSs are provided with sufficient electric energy and 30 charging slots through entire simulation, using the charging rate of $10 \mathrm{~kW}$ (using the constant charging power in our work can refer to many previous works on common CS-selection schemes e.g, [10]-[12], [17]). 30 fully charged batteries are initially set for each CS. This is different from previous works on demand response where the charging power is dynamically adjusted. Here, the shortest path towards CS is formed considering the Helsinki road topology.

Even if each EV reaching the SOC threshold, may request battery switch at different time slot due to its variable speed ranging between $[30 \sim 50] \mathrm{km} / \mathrm{h}$ and initial location, the charging management is essential as some EVs need to wait additional time for battery switch, until a battery is fully charged by CS and then becomes switchable. The following schemes are evaluated for comparison:

- Battery Switch (BS): The proposed centralized CSselection scheme in Section III-F based on the battery switch system, not bringing the EVs' reservations. This means the queue of $N_{R}$ is always 0 , as EV will not report its reservation. Besides, BS $(\mathbf{O})$ is as the way to estimate batteries availability in [17].

- Reservation-BS: The proposed centralized CS-selection scheme in Section III-F based on the battery switch system, with EVs' reservations enabled.

- A-Reservation-BS: The proposed decentralized CSselection scheme in Section IV, where EVs' reservations are delivered through anycasting way.

- Minimum Queuing Time (MQT) [5]: The centralized CS-selection scheme based on the plug-in charging technology [5], which selects the CS with the minimum queueing time.

- Reservation-1 [10], Reservation-2 [11]: The plug-in charging based centralized CS-selection schemes, by taking EVs' reservations into account. Note that in [10], the estimation is decoupled into 10 time intervals.

The simulation represents a 12 hours' duration with a $\gamma=$ $0.1 \mathrm{~s}$ resolution. So, the EVs positions, speeds and energies are updated every $0.1 \mathrm{~s}$, on the road or at a CS. The following performance metrics are evaluated:

- Average Waiting Time for Switch (AWTS): The average period between the time an EV arrives at the selected CS and the time it finishes battery switch, as the performance metric at $\mathrm{EV}$ side.

- Total Switched Batteries (TSB): The total number of EVs have been switched with batteries at CSs, as the charging performance metric at CS side.

- Total Reservations Making (TRM): The communication cost for reservation service, captured through the cellular network.

\section{A. Influence of Charging Power}

In Fig. 8(a), we observe the performance (in terms of AWTS and TSB) applying the STCF policy to charge depleted 
batteries, outperforms that applying the First Deplete First Charge (FDFC) policy. This is because that CSs will not experience a long service queue, if the period for batteries cycling is reduced via the STCF policy. Whereas in case of FDFC, the batteries which can be fully charged in short time may be delayed for charging, due to their later depleted time from EVs. In the following evaluation, we apply the STCF policy for battery cycling.

The advantage of applying the reservation service is reflected by comparing BS with Reservation-BS. Besides, both a less number of charging slots $\delta$ and batteries $N_{B}$, degrade performance. This is mainly due to the lack of switchable batteries for incoming EVs. This is because as less EVs' batteries are switchable at CSs, the time for other parked EVs to wait for battery switch increases. Furthermore, BS (O) performs worse than BS. This is due to the proposed scheme jointly considers the expected number of switch batteries, for balancing the switchable batteries among CSs.

If increasing the charging power at CSs, the performance is improved in Fig. 8(b) and Fig. 8(c) respectively. In particular, reservation-enabled scheme benefit more from increased charging power than other schemes. This implies that a fast charging power is able to service EVs towards a saturation, even not with the battery switch technology. Here, the benefit of enabling battery switch over plug-in charging system is reflected, by comparing "Reservation-BS" with "Reservation1" and "Reservation-2". Particularly, we observe that those with/without reservation service enabled, start to perform closely under $50 \mathrm{~kW}$ case. This implies that when incoming EVs, or depleted batteries can be fast recharged, the benefit of enabling EVs' reservations becomes subtle. In other words, most likely there will not be charging hotspot at CSs.

\section{B. Influence of Density of EVs}

Results in Fig. 9(a) and Fig. 9(b) show that, the battery switch system outperforms plug-in system, even in case of a lower EVs density. This is directly related to the contributions from battery cycling and proposed CS-selection scheme. Here, both the "Reservation-BS" and "BS" perform closely given 150 EVs. This is because that, the initially maintained $30 \times$ $7=210$ batteries is sufficient to support timely battery switch while without additional waiting.

As the number of EVs increases, enabling the reservation for CS-selection starts to show its benefit, by balancing the batteries switched as well as minimizing the time to wait for switchable batteries. In spite of this, the CS-selection schemes under the plug-in charging system ("Reservation1" and "Reservation-2") still performs worse than those ("Reservation-BS" and "A-Reservation-BS") under the battery switch system. Here, the decentralized "A-Reservation-BS" is with a slightly worse performance (a longer AWTS and less TSB), because of a periodical information broadcasting.

However, in Fig. 9(c), "A-Reservation-BS" achieves a much lower cost to deliver EV's reservations through the cellular network, compared to the centralized "A-Reservation-BS". This thanks to the $\mathrm{V} 2 \mathrm{~V}$ anycasting nature, to rely on the opportunistic vehicles encounters.

\section{Influence of CS Broadcasting Interval}

In Fig. 10(a) and Fig. 10(b), we observe that infrequent CS broadcasting $\Delta$ (e.g., 900s), degrades both AWTS and TSB under "A-Reservation-BS". This is mainly because of the obsolete information received by EVs, that leads to suboptimal CS-selection.

While, since other compared schemes are with centralized manner, they are not affected by $\Delta$. In Fig. 10(c), if decreasing the $\mathrm{V} 2 \mathrm{~V}$ transmission range, the case "A-Reservation-BS (100m)" suffers from much higher TRM. This is because the infrequent encounter between EVs, is unable to timely deliver reservation through anycast-driven $\mathrm{V} 2 \mathrm{~V}$ manner. As such, most of EV's reservations will be delivered through the cellular network as the back-up solution, at the time approaching the next broadcasting. With the default $300 \mathrm{~m}$ case (shown as 'AReservation-BS"), such cost is dramatically reduced, as more EVs' reservations can be delivered through V2V anycasting.

\section{Future Works}

If bringing the heterogeneous battery switch system, the difference of information to be required from depleted battery (of the EV on-the-move), is still the required charging time of battery. Such required charging time depends on the fully volume of battery (because the charging power at CS is not changed). As this work assumes EVs are with homogeneous batteries, future work will consider the compatibilities between heterogeneous EVs and batteries (e.g., each type of EV can only be switched with a certain type of battery).

Also, the battery degradation should be taken into account for CS-selection, concerning the impact of charging power and frequency etc. For example, for the comfort of EV drivers, they may prefer to switch the battery at a CS which fast cycles depleted batteries using a higher power. Whereas, this would bring a negative impact on the battery State of Health (SOH). Therefore, the trade-off between SOH and driver's comfort is worthwhile investigation.

\section{CONCLUSION}

In this article, we investigated the battery switch technology to enable fast EV charging in urban city. The system addresses the fast cycling policy to provide switchable batteries for incoming EVs. Also, EVs' reservations including arrival time and expected charging time of batteries, are taken into account to estimate the future status of CSs. The CS-selection policy follows the rules to balance the number of batteries switched among CSs, and to minimize time to wait for switch (if currently there is no battery switchable). Evaluation results under the Helsinki city scenario showed the advantage of our proposal CS-selection scheme, in terms of charging performance at EVs and CSs side. A decentralized system is provisioned to address some EVs' privacy concerns, outperforms other schemes in terms of communication cost for reservation service.

\section{REFERENCES}

[1] J. Mukherjee and A. Gupta, "A Review of Charge Scheduling of Electric Vehicles in Smart Grid," IEEE Systems Journal, vol. 9, no. 4, pp. 15411553, December, 2015. 


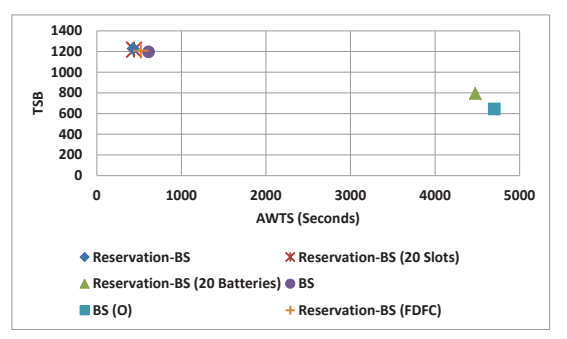

(a) AWTS v.s. TSB Given $10 \mathrm{~kW}$ Charging Power

Fig. 8. Influence of Charging Power $\beta$

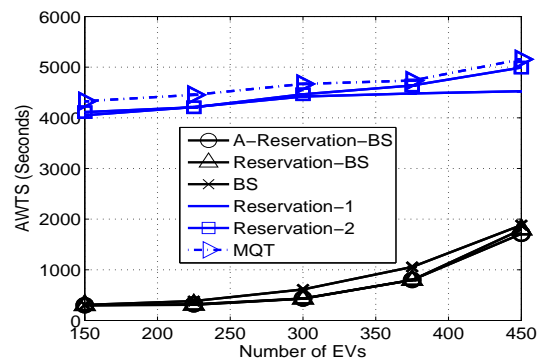

(a) AWTS

Fig. 9. Influence of EVs Density $N_{e v}$

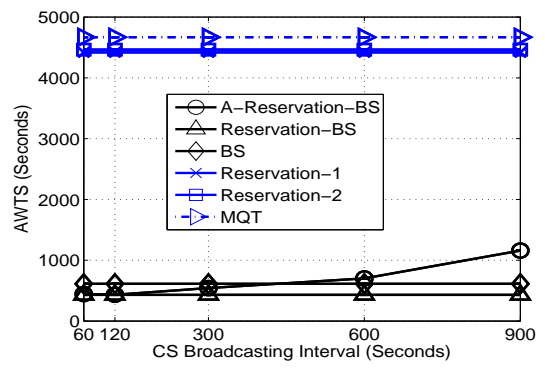

(a) AWTS

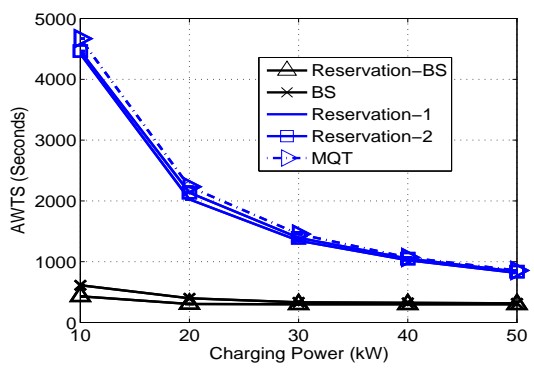

(b) AWTS

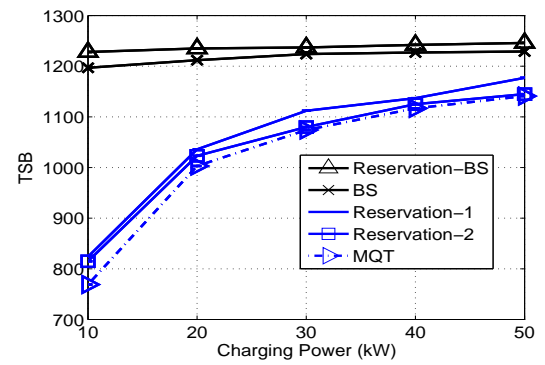

(c) TSB

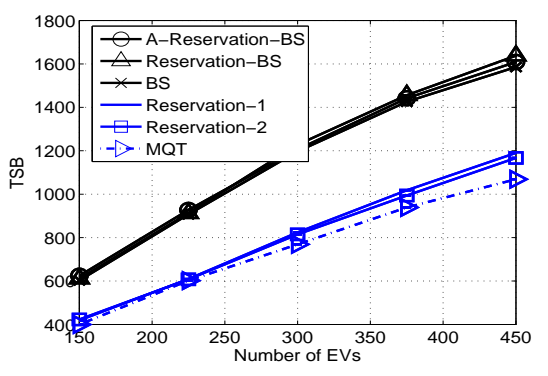

(b) TSB

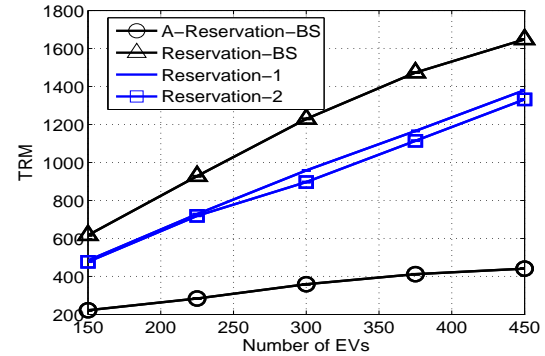

(c) TRM

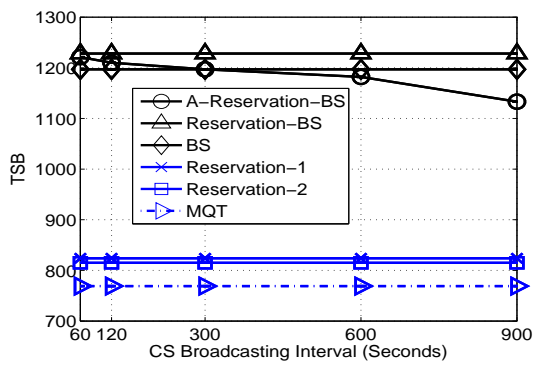

(b) TSB

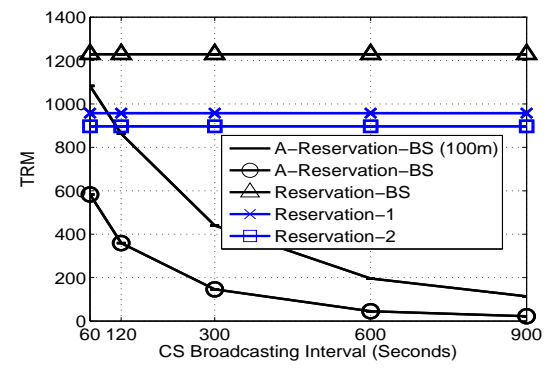

(c) TRM

Fig. 10. Influence of CS Broadcasting Interval $\Delta$

[2] E. Rigas, S. Ramchurn, and N. Bassiliades, "Managing Electric Vehicles in the Smart Grid Using Artificial Intelligence: A Survey," IEEE Transactions on Intelligent Transportation Systems, vol. 16, no. 4, pp. 1619-1635, August, 2015.

[3] M. Gharbaoui, L. Valcarenghi, R. Bruno, B. Martini, M. Conti, and P. Castoldi, "An Advanced Smart Management System for Electric Vehicle Recharge," in IEEE IEVC' 2012, Greenville, SC, USA, March, 2012.

[4] F. Hausler, E. Crisostomi, A. Schlote, I. Radusch, and R. Shorten, "Stochastic Park-and-Charge Balancing for Fully Electric and Plugin Hybrid Vehicles," IEEE Transactions on Intelligent Transportation Systems, vol. 15, no. 2, pp. 895-901, April, 2014.

[5] Y. Cao, N. Wang, and G. Kamel, "A Publish/Subscribe Communication Framework For Managing Electric Vehicle Charging," in IEEE ICCVE' 14, Vienna, Austria, November, 2014.

[6] E. Rigas, S. Ramchurn, N. Bassiliades, and G. Koutitas, "Congestion Management for Urban EV Charging Systems," in IEEE SmartGridComm '13, Vancouver, Canada, October, 2013.

[7] S.-N. Yang, W.-S. Cheng, Y.-C. Hsu, C.-H. Gan, and Y.-B. Lin, "Charge Scheduling of Electric Vehicles in Highways," Elsevier Mathematical and Computer Modelling, vol. 57, no. 11?2, pp. 2873 - 2882, June, 2013.

[8] F. Malandrino, C. Casetti, C.-F. Chiasserini, and M. Reineri, "A gametheory analysis of charging stations selection by ev drivers," Performance Evaluation, vol. 83-84, no. Supplement C, pp. 16 - 31, 2015.

[9] M. M. de Weerdt, E. Gerding, S. Stein, V. Robu, and N. R. Jennings,
"Intention-Aware Routing to Minimise Delays at Electric Vehicle Charging Stations," in AAAI' 13, Bellevue, Washington, USA, July, 2013.

[10] Y. Cao, O. Kaiwartya, R. Wang, T. Jiang, Y. Cao, N. Aslam, and G. Sexton, "Towards Efficient, Scalable and Coordinated On-the-move EV Charging Management," IEEE Wireless Communications, vol. 24, no. 2, pp. 66-73, April, 2017.

[11] Y. Cao, N. Wang, G. Kamel, and Y.-J. Kim, "An Electric Vehicle Charging Management Scheme Based on Publish/Subscribe Communication Framework," IEEE Systems Journal, vol. 11, no. 3, pp. 1822-1835, 2017.

[12] H. Qin and W. Zhang, "Charging Scheduling With Minimal Waiting in a Network of Electric Vehicles and Charging Stations," in ACM VANET ' 11, Las Vegas, Nevada, USA, September, 2011.

[13] Y. Cao, T. Wang, O. Kaiwartya, G. Min, N. Ahmad, and A. H. Abdullah, "An EV Charging Management System Concerning Drivers' Trip Duration and Mobility Uncertainty," IEEE Transactions on Systems, Man, and Cybernetics: Systems, Accepted in 2016.

[14] T. Winkler, P. Komarnicki, G. Mueller, G. Heideck, M. Heuer, and Z. Styczynski, "Electric Vehicle Charging Stations in Magdeburg," in IEEE VPPC '09, Dearborn, Michigan, September, 2009.

[15] F. Pan, R. Bent, A. Berscheid, and D. Izraelevitz, "Locating PHEV Exchange Stations in V2G," in IEEE SmartGridComm' 10, Maryland, USA, October 2010.

[16] X. Tan, B. Sun, and D. H. K. Tsang, "Queueing network models for electric vehicle charging station with battery swapping," in 2014 IEEE International Conference on Smart Grid Communications (SmartGridComm), November 2014, pp. 1-6.

[17] Y. Cao, S. Yang, G. Min, X. Z. ang Houbing Song, O. Kaiwartya, and 
N. Aslam, "A Cost-Efficient Communication Framework For Battery Switch Based EV Charging," IEEE Communications Magazine, vol. 55, no. 5, pp. 162-169, May, 2017.

[18] O. Kaiwartya, A. H. Abdullah, Y. Cao, A. Altameem, M. Prasad, C. T. Lin, and X. Liu, "Internet of vehicles: Motivation, layered architecture, network model, challenges, and future aspects," IEEE Access, vol. 4, pp. 5356-5373, 2016.

[19] Y. Cao, Z. Sun, N. Wang, H. Cruickshank, and N. Ahmad, "A Reliable and Efficient Geographic Routing Scheme for Delay/Disruption Tolerant Networks," IEEE Wireless Communications Letters, vol. 2, no. 6, pp. 603-606, December, 2013.

[20] M. T. Beck, M. Werner, S. Feld, and S. Schimper, "Mobile edge computing: A taxonomy," in Proc. of the Sixth International Conference on Advances in Future Internet, November 2014.

[21] Y. Cao and Z. Sun, "Routing in Delay/Disruption Tolerant Networks: A Taxonomy, Survey and Challenges," IEEE Communications Surveys Tutorials, vol. 15, no. 2, pp. 654-677, Second Quarter, 2013.

[22] F. Kargl, P. Papadimitratos, L. Buttyan, M. Muter, E. Schoch, B. Wiedersheim, T.-V. Thong, G. Calandriello, A. Held, A. Kung, and J.-P. Hubaux, "Secure Vehicular Communication Systems: Implementation, Performance, and Research Challenges," IEEE Communications Magazine, vol. 46, no. 11, pp. 110-118, November, 2008.

[23] A. Keränen, J. Ott, and T. Kärkkäinen, "The ONE Simulator for DTN Protocol Evaluation," in ICST SIMUTools '09, Rome, Italy, March, 2009.

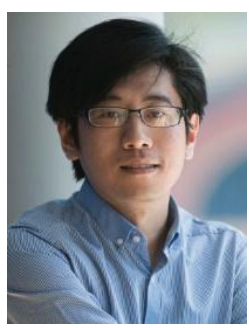

Yue Cao received the $\mathrm{PhD}$ degree from the Institute for Communication Systems (ICS), at University of Surrey, Guildford, UK in 2013. He was a Research Fellow at the ICS until September 2016, and Lecturer in Department of Computer and Information Sciences, at Northumbria University, Newcastle upon Tyne, UK until July 2017, and the Senior Lecturer since August 2017. His research interests focus on DTNs, E-Mobility, QoS/QoE in 5G. He is the Associate Editor of IEEE Access.

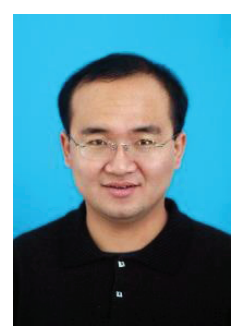

Wang Tong is an Associate Professor at Information and Communication Engineering College, Harbin Engineering University, China. He received $\mathrm{PhD}$ degree in Computer Application from Harbin Engineering University in 2006. His research interests include Wireless Sensor Networks (WSNs), Vehicular Ad-Hoc Networks (VANETs) and Internet of Things (IoT).

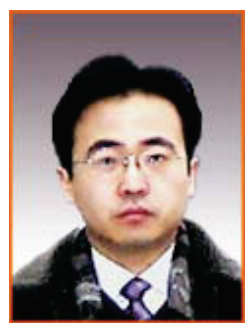

Xing Zhang is a Professor with the School of Information and Communications Engineering, Beijing University of Posts and Telecommunications, China. His research interests are mainly in satellite network$\mathrm{s}$, mobile edge computing,big data and Internet of Things.

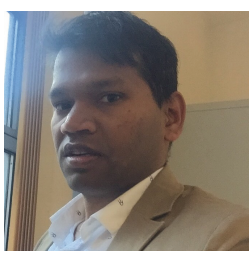

Omprakash Kaiwartya received his $\mathrm{PhD}$ degree in Computer Science from School of Computer and Systems Sciences, Jawaharlal Nehru University, New Delhi, India in 2015. He is currently a Research Associate at the Department of Computer and Information Sciences, Northumbria University, UK. He was Postdoctoral Research Fellow at the Faculty of Computing, Universiti Teknologi Malaysia, Johor Bahru, Malaysia. His research interests focus on Internet of connected Vehicles, EV charging, and IoT use cases in Sensor Networks.

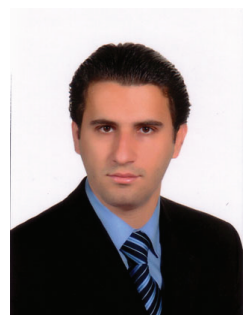

Things.
Mahmoud Hashem Eiza is a Lecturer in Computing (Computer and Network Security) at the School of Physical Sciences and Computing, University of Central Lancashire. Prior to that, he was a researcher in network security and privacy in the Department of Computer Science at Liverpool John Moores University (LJMU). His research interests include computer, communication, and network security, with specific interests in quality-of-service and wireless network security and privacy in vehicular Networks, Smart Grids, Cloud Computing, and Internet of

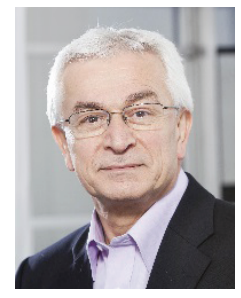

Ghanim Putrus leads the Power and Wind Energy Research group at the Faculty of Engineering and Environment, Northumbria University. He is Associate Editor for Elsevier Renewable Energy journal and serves on the technical/steering committees for several conferences. He is involved in the Institution of Engineering Technology (IET) professional activities and served on the executive committee of the IET Power Trading and Control Professional Network (2001-2009). His main research interests are the application of power electronics in power systems, power quality, integration of renewable energy resources and electric vehicles into power distribution networks. 